\title{
Security Warnings for Children's Smart Phones: A First Design Approach
}

\author{
Jana Fruth, Ronny Merkel, and Jana Dittmann \\ Otto-von-Guericke University Magdeburg, P.O. Box 4120, 39016 Magdeburg, Germany \\ fruth, merkel, dittmann\}eovgu.de
}

\begin{abstract}
In this paper we introduce a first design approach for security warnings on smart phones for children, based on recommendations of a paediatrician expert. To ease the understanding of malware threats and facilitate a adequate handling, children friendly design principles and simple descriptions are used. Amongst visual information, acoustical and haptic information are used to raise children's attention to the warning. Currently, a first implemented prototype of security warnings is tested with primary school students.
\end{abstract}

\section{Problem}

Modern mobile phones, so called "smart phones" are a part of our daily lives. According to a German survey [1], nearly $82 \%$ of children between 10 and 12 years possess an own mobile phone. These personal devices are often in the focus of attackers because of the storage of person related data, such as address books, which are worthwhile targets. To realise remote attacks on smart phones, attackers often use malicious codes. Today, more than 100 different malicious codes for mobile phones are known [3]. Certain malware attacks on children are not published presently. But above mentioned malware could also be used to attack smart phones of children. Anti-malware programs could protect mobile phones against these malware threats. Today, these security applications inform users with security warnings for malware infections of the mobile phone. In our opinion, these security warnings are designed for standard users and so they are not adequately adapted for specific user groups, which could differ in skills, dependending on user's age, profession, or health. We think, the adaptation of security warnings to childrens skills could help to sensitise them to malware threats on their smart phones and to train them playfully in right handling of anti-malware programs. In this paper, we introduce a first design approach for multi-media security warnings for smart phones used by children. Currently, a first implementation on an iPhone is tested with primary school students (age between 8 to 9 years).

\section{Solution}

Our concept for a design of security warnings for smart phones is inspired by different preliminary works from different application domains, like automotive domain [4] and automated production domain [2]. Our warning message design approach should fulfil two main requirements: the adaptation to children's skills and adaptation to requirements of smart phones. To realise children friendly security warnings, we follow the

B. de Decker et al. (Eds.): CMS 2011, LNCS 7025, pp. 241-243, 2011.

(c) IFIP International Federation for Information Processing 2011 
recommendations of a paediatrician expert and design the warnings by using elements which children know from their infantile experience and imaginary world. So, we apply characters known from computer games or comics, to express threat levels, for example through the character's mimic or colours (see figure 1). Furthermore, security program functions like the deletion of malicious codes are playfully communicated. For example, to quarantine a malware the child has to arrest a monster in prison (see figure 2). A feedback information about the success of the action is given. Additionally, a simple action chain is used to communicate malware threats and countermeasures to children. First: warning the user about current malware on his/her smart phone, second: explaining the risk level of the malware, third: recommending of security measures. Following the paediatrician expert's recommendation, multi-media stimuli are use to facilitate the children's learning. Therefore, the warnings include visual (different colours, simple textual descriptions), acoustical (warn signals, spoken descriptions, sounds) and tactile information (vibration). Our security warnings are adapted to the properties of smart phones, like limited size of display, limited audio quality and possibilities to interact with them.
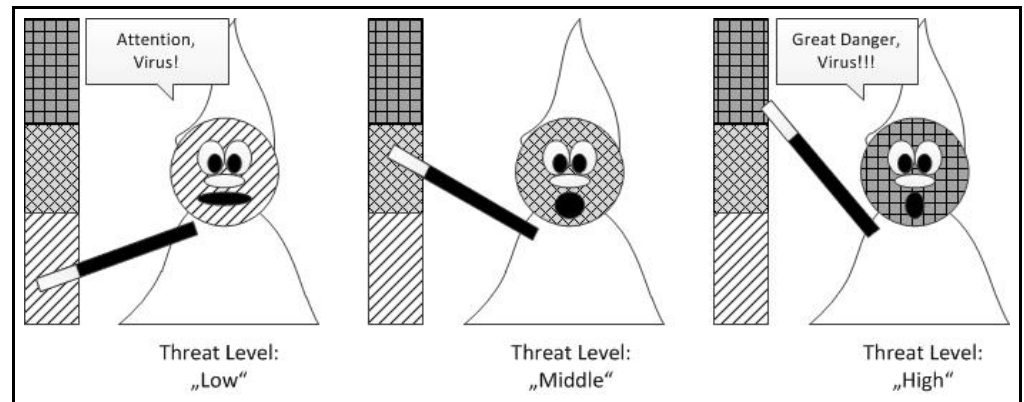

Fig. 1. Example of security alert character and visualisation of security threat levels (Remark: in the implementation the hachured regions are replaced by colours)

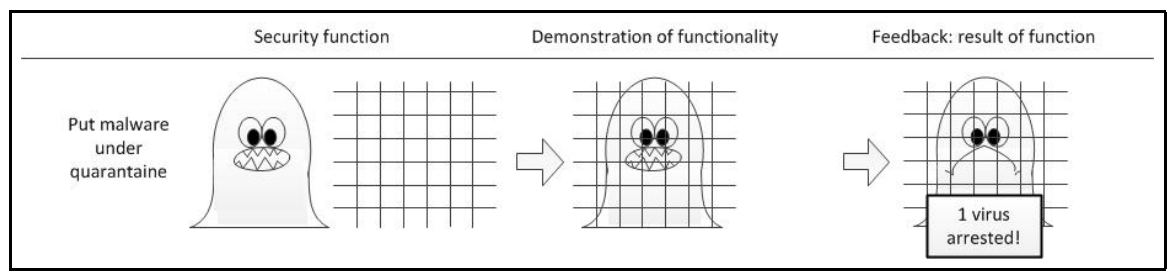

Fig. 2. Exemplary function of a security program on children's smart phones

\section{Future Work}

In the future, our first design approach has to be specified and realised on different mobile devices and evaluated with infantile test persons. Currently, an implementation of 
our security warning design approach on an iPhone is tested and evaluated with primary school pupils in the age between 8 and 9 years. The enhancement and evaluation of the sequence of warning, information and instructions, variations of threat levels, presented multi-media information, and different characters for security guides or malicious codes are also necessary. Furthermore, it is very interesting to evaluate, how interand intra-individual variability could have an influence on children's perception. Also, our design approach for security warnings could be adapted and used on other embedded devices, e.g. game robots. Another interesting question is, the design of security warnings and user instructions for disabled people using embedded devices, like smart phones or intelligent service robots.

Acknowledgement. We want to thank Prof. Dr. med. Jorch, PhD Hinz, PhD Herper, and Wiebke Menzel. The work of Jana Fruth is funded by the German Ministry of Education and Science (BMBF), project 01IM10002A. The presented work is part of the ViERforES1 project.

\section{References}

1. Bundesverband Informationswirtschaft, Telekommunikation und neue Medien e.V (BITKOM). Jugend 2.0: Eine repräsentative Untersuchung zum Internetverhalten von 10- bis 18-Jährigen (2011)

2. Fruth, J., Krätzer, C., Dittmann, J.: Design and Evaluation of Multi-Media Security Warnings for the Interaction between Humans and Industrial Robots. In: IS\&T / SPIE Electronic Imaging (2011)

3. German Federal Office for Information Security (BSI). Mobile Endgeräte und mobile Applikationen: Sicherheitsgefährdungen und Schutzmaßnahmen (2006)

4. Tuchscheerer, S., Dittmann, J., Hoppe, T., Krems, J.: Theoretical analysis of security warnings in vehicles and design challenges for the evaluation of security warnings in virtual environments. In: International Workshop on Digital Engineering (IWDE 2010), Magdeburg, pp. 33-37 (2010)

${ }^{1}$ www.vierfores.de 